\title{
Moth-Eaten Symptomatic Syphilitic Alopecia Associated with Human Immunodeficiency Virus-A Case Report
}

\author{
Tomohisa Fukui 1,2*, Yusuke Sakuraba', Ken Harada², Hajime Nakano', Daisuke Sawamura1 \\ ${ }^{1}$ Department of Dermatology, Hirosaki University Graduate School of Medicine, Hirosaki, Japan \\ ${ }^{2}$ Department of Dermatology, Aomori Central Hospital, Aomori, Japan \\ Email: *derma@hirosaki-u.ac.jp
}

Received 31 March 2016; accepted 24 May 2016; published 27 May 2016

Copyright @ 2016 by authors and Scientific Research Publishing Inc.

This work is licensed under the Creative Commons Attribution International License (CC BY). http://creativecommons.org/licenses/by/4.0/

(c) (i) Open Access

\begin{abstract}
Syphilitic alopecia (SA) is a rare skin manifestation in secondary syphilis and sometimes indistinguishable from other alopecia. SA is of 2 types, symptomatic and essential, and 3 clinical types, moth-eaten or patchy pattern, diffuse pattern and a combination of both. SA in our case indicated symptomatic SA with moth-eaten hairloss. A 46-year-old Japanese homosexual man experienced hairloss followed by the pruritic skin rash. Physical examination of the scalp showed diffusely spread papulosquamous erythema and incomplete hairloss around the lesions. The scalp lesions appeared as "moth-eaten alopecia". Serological examination for syphilis and HIV were positive. Thus, SA complicated with HIV infection was diagnosed. He was treated with a single perioral dose of ampicillin for 4 weeks, then his condition improved rapidly and hair regrowth occurred. HIVinfected persons show a high incidence of syphilis. Therefore, HIV test should be considered for patients with alopecia.
\end{abstract}

\section{Keywords}

Alopecia, Syphilis, Syphilitic Alopecia, Moth-Eaten Alopecia, HIV

\section{Introduction}

Syphilis and HIV are a sexually transmitted infection and the number of the cases complicated by them is demonstrated to increase recently. Syphilis presents various manifestations indistinguishable from other skin diseases. Among them, SA is a rare skin manifestation in secondary syphilis with an incidence of $2.9 \%-11.2 \%$ [1] [2].

${ }^{*}$ Corresponding author.

How to cite this paper: Fukui, T., Sakuraba, Y., Harada, K., Nakano, H. and Sawamura, D. (2016) Moth-Eaten Symptomatic Syphilitic Alopecia Associated with Human Immunodeficiency Virus-A Case Report. Case Reports in Clinical Medicine, 5, 188-190. http://dx.doi.org/10.4236/crcm.2016.55035 
It is classified into a few types and is sometimes misdiagnosed. We present the case of a 46-year-old Japanese homosexual man with moth-eaten symptomatic SA complicated by HIV infection.

\section{Case Report}

A 46-year-old Japanese homosexual man has moth-eaten symptomatic SA. The patient experienced hairloss since a week before he was referred to our department. He had sexual intercourse with a male partner 4 weeks before his referral. He did not have a history of genital chancre until then. Physical examination of the scalp showed diffusely spread papulosquamous erythema and incomplete hairloss around the lesions. The hair-pull test result was negative. The scalp lesions appeared as "moth-eaten alopecia" (Figure 1(a)). Trichoscopy revealed black dots, telangiectasia, but not exclamation mark hair. Furthermore, pruritic papulosquamous erythema on the trunk and proximal limbs appeared approximately 3 weeks before the onset of hairloss (Figure 1(b)). The patient presumed that these symptoms were caused by atopic dermatitis that persisted since his childhood. Headache, ocular symptoms, or neural symptoms were absent.

Serological screening for syphilis by using the Rapid Plasma Reaction (RPR) test showed increased titers (208 U/mL), and the results of treponema pallidum hemagglutination assay and fluorescent treponemal antibody-absorption test were positive. The serological test result for anti-human immunodeficiency virus-1 (HIV-1) antibody and polymerase chain reaction results for HIV-1 RNA were positive. CD4 population was decreased to $457 / \mu \mathrm{L}$ (normal range: $700-1500 / \mu \mathrm{L}$ ).

Thus, SA complicated with HIV infection was diagnosed. He was treated with a single peroral dose of ampicillin (3000 mg/d) for 4 weeks, after which his condition improved rapidly and hair regrowth occurred (Figure 1(c)). Finally, 30 weeks after the treatment, the titer in the RPR test decreased to $8.0 \mathrm{U} / \mathrm{mL}$, and relapse did not occur. His HIV infection had been followed closely without treatment, because the loss of CD4 cells was slight to moderate.

\section{Discussion}

\subsection{Classification of SA}

SA is a rare skin manifestation in secondary syphilis and occurs in approximately $4 \%-12.5 \%$ of patients with syphilis [1]. According to McCarthy, SA is of 2 types: symptomatic and essential [3]. The former type is associated with other syphilitic lesions, and the latter is not associated with any other syphilitic mucocutaneous lesions. Essential SA is sometimes misdiagnosed such as alopecia areata and trichotillomania because of the absence of any other mucocutaneous lesions [4]. Moreover, clinical types were classified as follows: 1) moth-eaten or patchy pattern presenting as small scattered plaques of non-scarring and incomplete hairless alopecia, 2) diffuse pattern caused by widespread telogen effluvium-type hairloss, and 3) a combination of both [5]. Our patient showed patchy moth-eaten hairloss and other secondary syphilitic lesions on the scalp and elsewhere. Therefore, SA in our case indicated symptomatic SA with moth-eaten hairloss.
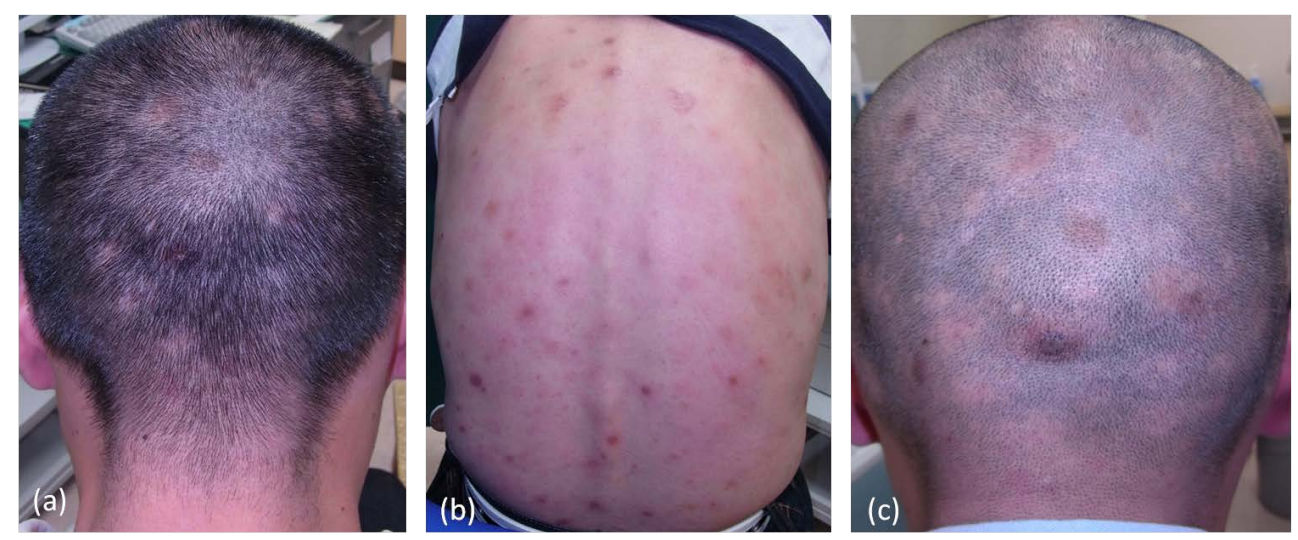

Figure 1. Clinical findings. (a) Moth-eaten alopecia spread on the scalp, around the papulosquamous lesions. (b) Papulosquamous lesions on the trunk and proximal limbs. (c) Marked improvement observed during follow-up at 4 weeks after the treatment. 


\subsection{Association with HIV}

HIV-infected persons show a high incidence of syphilis, probably because the incidence of HIV infection in patients with syphilis is high [6]. Therefore, HIV test should be performed for all patients with syphilis. Dermatologists can play important roles in SA diagnosis. Thus, we present a case of moth-eaten symptomatic SA. Serologic tests for syphilis and HIV infection are recommended for patients with hairloss.

\section{Conclusion}

We present a case of moth-eaten symptomatic SA. SA is a rare skin manifestation in secondary syphilis and sometimes misdiagnosed. HIV-infected persons show a high incidence of syphilis. Thus serologic tests for syphilis and HIV infection are recommended for patients with hairloss.

\section{Conflict of Interest}

The authors have no conflict of interest.

\section{References}

[1] Vafaie, J., Weinberg, J.M., Smith, B. and Mizuguchi, R.S. (2005) Alopecia in Association with Sexually Transmitted Disease: A Review. Cutis, 76, 361-366.

[2] Hira, S.K., Patel, J.S., Bhat, S.G., Chilikima, K. and Mooney, N. (1987) Clinical Manifestations of Secondary Syphilis. International Journal of Dermatology, 26, 103-107. http://dx.doi.org/10.1111/j.1365-4362.1987.tb00532.x

[3] Hernández-Bel, P., Unamuno, B., Sánchez-Carazo, J.L., Febrer, I. and Alegre, V. (2013) Syphilitic Alopecia: A Report of 5 Cases and a Review of the Literature. Actas Dermo-Sifiliográficas, 104, 512-517. http://dx.doi.org/10.1016/j.ad.2012.02.009

[4] Bi, M.Y., Cohen, P.R., Robinson, F.W. and Gray, J.M. (2009) Alopecia Syphilitica Report of a Patient with Secondary Syphilis Presenting as Moth-Eaten Alopecia and a Review of Its Common Mimickers. Dermatology Online Journal, 15, 6 .

[5] Cuozzo, D.W., Benson, P.M., Sperling, L.C. and Skelton, H.G. (1995) Essential Syphilitic Alopecia Revisited. Journal of the American Academy of Dermatology, 32, 840-843. http://dx.doi.org/10.1016/0190-9622(95)91543-5

[6] Chesson, H.W., Heffelfinger, J.D., Voigt, R.F. and Collins, D. (2005) Estimates of Primary and Secondary Syphilis Rates in Persons with HIV in the United States, 2002. Sexually Transmitted Diseases, 32, 265-269.

http://dx.doi.org/10.1097/01.olq.0000162359.75509.9c 